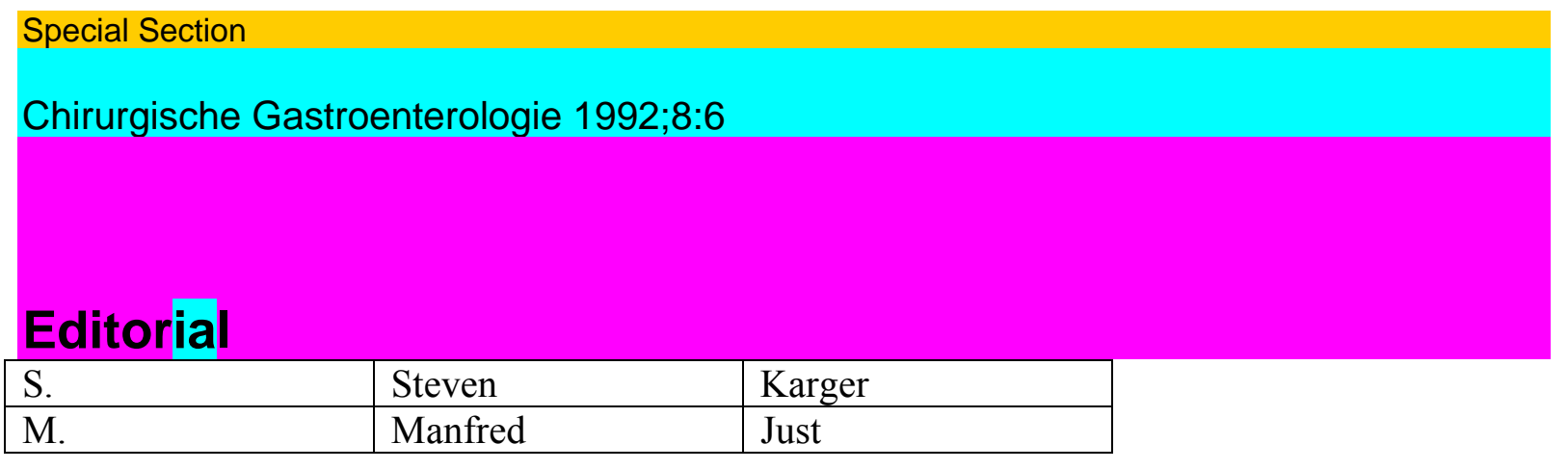

Basel, Germering

Liebe Leserin, lieber Leser

Was Sie in der Hand halten, ist nicht schon wieder ein Heft einer neuen Zeit-schrift, sondern eine bewährte und be-standene Publikation in neuer Form. Die Übernahme der Verlagsrechte des TM-Verlages durch den Verlag S. Karger erfolgte allein aus Altersgründen des Gründers und Inhabers, Prof. C. E. Zöckler. Wir freuen uns, eine qualitativ hochstehende und in alien Kreisen anerkannte Zeitschrift übernehmen zu dürfen und werden versuchen, sie noch auf eine breitere Basis zu stellen. Die Tatsache, daß die «Chirurgische Gastroenterologie mit interdisziplinären Ge-sprächen» sich nahtlos in unser Zeit-schriftenprogramm einfügt, bildet eine gute Voraussetzung dazu. Mit der neuen Schriftleitung - beste-hend aus dem Hauptschriftleiter, Professor Paquet - und den Schriftleitern, Professor Bodner, Professor Horn, Professor Säuberli, sind wir sicher, ein Team gefunden zu haben, das das außeror-

dentlich hohe Niveau der Redaktion auch in Zukunft gewährleisten wird. An der Konzeption der Zeitschrift mit den Bereichen «Eingeladene Beiträge», «lnterdisziplinäre Gespräche», «Redaktionsteil» und «Originalarbeiten» wird sich in Zukunft nichts ändern. Der Verlag sichert Ihnen als Leser zu, daß Sie Ihre «Chirurgische Gastroenterologie» pünktlich jeweils in der letzten Quar-talswoche erhalten. Des weiteren kön-nen wir Ihnen heute sehr interessante Supplementhefte für das Jahr 1992 avisieren. Die positive Resonanz der Leserschaft veranlaßt den Verlag, die Zeitschrift in einer IVW-geprüften Auf-lage von 4000 Exemplaren im deutschsprachigen Raum zu vertreiben. Für das Vertrauen aller Beteiligten in unseren Verlag danken wir und freuen uns auf eine gute, fruchtbare und erfolg-reiche Zusammenarbeit und wünschen Ihnen, liebe Leser, eine stets interessante und informative Lektüre. Konstruk-tive Kritik wie auch Anregungen sind uns immer willkommen.

Karger/Just

Editorial

ASCHE AG, Hamburg, informiert

(R)

\title{
GASTRAX
}

Ein H2-Blocker

der neueren Generation mit dem Wirkstoff Nizatidin.

Ausgezeichnete klinische Wirksamkeit

Gute Kombinierbarkeit mit anderen Pharmaka

Einfach in der Anwendung 
Gastrax ${ }^{\circledR} /$ Gastrax ${ }^{\circledR}$ mite Zusammen-setzung: 1 Kapsel Gastrax/Gastrax mite enthält 300/150 mg Nizaiidin Anwendungsgebiete: Zur Behand-lung von Erkra $\pi$ kungen im oberen Magen-DarmBereich, bei denen eine Verminderung der Magensäuresekre-tion angezeigt ist, wie Duodenalulzera, benigne Magenulzera. Gastrax mite au ßerdem zur Rezidivprophylaxe von Duodenalulzera. Gegenanzeigen: Überempfindlichkeitgegen H -Rezep-tor-Antagonisten. Maligne Magenulzera vor der Behandlung ausschlie-ßen. Kinder, da Verträglichkeit und Wirksamkeit nicht klinisch geprüft. Schwangerschaft, geplante Schwan-gerschaft, da klinische Erfahrungen nicht vorliegen. Tierversuche erga-ben keine Hinweise auf Fruchtschä-digungen. Stillzeit, da der Wirkstoff in die Muttermilch übergeht. Gastrax (300 mg Nizatidin) bei schwerer Nie-reninsuffizienz (Kreatinin-Clearance $<40 \mathrm{ml} / \mathrm{min}$ bzw. Serumkreati in-Kon-zentration $>$ $2,5 \mathrm{mg} / 100 \mathrm{ml})$ und bei Patienten über75 Jahre. Gastrax mite (150 mg Nizatidin) nicht zur Rezidivprophylaxe bei schwerer Nierenin-suffizienz (KreatininClearance $<40 \mathrm{ml} / \mathrm{min}$ bzw. Serumkreatinin-Konzentra-tion $>2,5 \mathrm{mg} / 100 \mathrm{ml}$ ) und bei Patienten über 75 Jahre. Hinweis: Bei Patienten mit Leberfunktionseinschrän-kung ist Vorsicht geboten. Nebenwirkungen: Gelegentlich Schläfrigkeit, Schwitzen, Kopfschmerzen, Schwin-del, Asthenie, Brustschmerzen, Myal-gie, ungewöhnlicheTräume, Schnup-fen, Entzündungen der Rachenschleimhaut, Husten, Juckreiz. Sel-ten: Überempfindlichkeitsreaktionen (z. B. Urticaria, Exanthem, ödematöse Schwellung, Larynxòdem, Broncho-spasmen, Anaphylaxie, Eosinophilie), exfoliative Dermatitis. In Einzelfällen: Gynäkomastie, Thrombozytopenie, thrombozytopenische Purpura, ..Ver-wirrtheitszustände, Fieber und Übel-keit sowie Potenzstörungen und ver-minderte Libido. Bei einigen Patienten vorübergehende Erhöhungen der Leberenzymwerte SGOT, SGPT und deralkalischen Phosphatase. Selten: SGOT und SGPT > 500 I. E./1 sowie Erhöhung des Bilirubin mit histo-morphologischen Veränderungen der Leber. Diese Erscheinungen bilden sich nach Absetzen des Arzneimittels spontan zurück. Erhöhung der Harn-säure- und Serumkreatininwerte. Blut-konzentrations-Veränderungen von Dihydrotestosteron, deren klinische Bedeutung noch unklar ist. Eine sym-ptomlose ventrikuläreTachykardie ist $\mathrm{m}$

\section{ASCHE AG}

\section{Arzneimitt $\theta 1$ mit Service G A S T R O}

wie auch bei unbehandelten Patienten - in Einzelfällen beobachtet wor-den. Wechselwirkungen: Bei gleich-zeitiger Gabe von sehr hohen Acetyl-salicylsäure-Dosen $(3900 \mathrm{mg}$ ) Erhöhung der ASS-Spiegel. Urobilinogen-Tests mit Multistix können falsch posi-tiv ausfallen.

Dosierungsanleitung/ Dauer der Anwendung: Ulcera duo-deni, Ulcera ventriculi: Gastrax $1 \mathrm{x}$ täg-lich abends 1 Kapsel; Gastrax mite $1 \mathrm{x}$ täglich 2 Kapseln (abends) bzw. 2x täglich (morgens und abends) 1 Kapsel. 4-8 Wochen je nach Behandlungs-erfolg. Rezidivprophylaxe: Gastrax mite 1x täglich abends 1 Kapsel; bis zu einem Jahr. Patienten mit schwerer Niereninsuffizienz (Kreatinin-Clearance $<40 \mathrm{ml} / \mathrm{min}$ bzw. Serumkreatinin-Konzentration $>2,5 \mathrm{mg} / 100 \mathrm{ml}$ ) und Patienten über 75 Jahre bei Ulcera duodeni/ventriculi 1 x täglich abends 1 Kapsel Gastrax mite. Packungs-größen und Preise: Gastrax Kapseln mit 300 mg Nizatidin: 20 (N1) - DM 119,60; 50 (N2) - DM 283,95; 100 (N3) - DM 555,00. Gastrax mite Kapseln mit 150 mg Nizatidin: 20 (N1) -DM 65,00; 50 (N2) - DM 150,35; 100 (N3) - DM 295,00. Weitere Einzelhei-ten enthalten die wissenschaftliche Broschüre und die Gebrauchsinfor-mation für Fachkreise. ASCHE AG. Postfach 5001 32, 2000 Hamburg 50. Stand: Mai 1991 